\title{
¿Es necesario resecar siempre las recidivas de los tumores vesicales superficiales en el momento del diagnostico?: comunicación preliminar
}

\author{
P. Martínez Cáceres, J.G. Hidalgo Arroyo, G.E. Chéchile Toniolo \\ Departamento de Urología. Instituto Médico Tecnológico. Barcelona.
}

Actas Urol Esp 2005; 29 (6): 567-571

\section{RESUMEN}

¿ES NECESARIO RESECAR SIEMPRE LAS RECIDIVAS DE LOS TUMORES VESICALES SUPERFICIALES EN EL MOMENTO DEL DIAGNÓSTICO?: COMUNICACIÓN PRELIMINAR

Introducción: Valorar la conducta expectante en las recidivas del tumor vesical superficial (TVS) mediante la observación post-diagnóstico y su implicancia clínica.

Material y métodos: De 112 pacientes diagnosticados de TVS, entre 1/1998 y 12/2003, se manejó con observación a 13, luego del diagnóstico cistoscópico de recidiva, hasta la decisión de tratamiento de las mismas.

Los controles se realizaron con cistoscopia flexible cada 3 meses. En los 13 pacientes se contabilizaron 15 eventos de observación ( 2 veces en 2 pacientes).

La decisión de observar y/o resecar se basó en la variación del aspecto, tamaño o número de los tumores y en la presencia o no de síntomas.

Se analizaron las características clínico-patológicas, y la evolución de las mismas.

Resultados: La edad media fue de 74,6 años (rangos 47-91). El tiempo medio de observación fue de 5,76 meses (rangos 3-17).

Entre los tumores previos a la observación y los post-observación, de los 15 eventos, en 12 que representa el $80 \%$ no hubo progresión en grado o estadio. En los 3 restantes se constató progresión en grado y estadio en 2 y sólo de estado en 1.

En ningún caso hubo progresión a tumor infiltrante ni aparición de tumor en TUS.

Conclusión: Por mínima progresión, ninguna a tumor infiltrante y escasa clínica, las recidivas podrían ser manejadas durante un tiempo con observación, disminuyendo la morbilidad y el coste asociados a la RTU.

Palabras clave: Tumor vesical superficial. Tumor vesical infiltrante. Tumor de tramo urinario superior.

\section{ABSTRACT}

IS IT NECESSARY TO TREAT ALWAYS THE RELAPSES OF SUPERFICIAL BLADDER TUMOUR AT THE MOMENT OF DIAGNOSIS?: PRELIMINARY COMMUNICATION

Objective: To asses the expectant management superficial recurrent bladder tumours.

Material and methods: Between Jan 1998 and Dec 2003, 112 patients were diagnosed of superficial bladder tumour. 13 patients were observed after the diagnosis of tumour relapse until the decision to perform a transurethral resection. We analysed the clinical and pathological features. The patients were controlled with flexible cystoscopy every 3 months approximately. Of the 13 patients we count 15 observation events, $(2$ times in 2 patients).

The decision between to treat or continue with observation was based in changes of appearance, size or number of tumours and presence of hematuria.

Results: With a mean age of 74,6 years (47-91). The mean observation time was 5,76 months (3-71) .

In the group of tumours previous to the observation period, in 12 events (10 patients) that means $80 \%$ any progression in grade or stage was observed. In the other 3 events we observed progression in grade and stage in 2 and only stage in 1 . In any case we observed progression to invasive bladder tumour neither upper urinary tract tumour.

Conclusions: Due to the low risk of progression, we believe that immediate eradication is not always necessary, thus reducing the adverse impact of repeat resections.

Keywords: Superficial bladder tumour. Observation. Recurrence. 
$\mathrm{E}$ 1 tratamiento habitual de los tumores vesicales superficiales, tanto iniciales como recidivados es la resección transuretral, sola o acompañada de biopsias múltiples randomizadas, de acuerdo al protocolo existente en cada centro, adecuadas a las condiciones o disponibilidad de los mismos, pero estandarizadas siempre de acuerdo al consenso internacional.

Es conocida desde hace décadas las complicaciones intra y post-operatorias inmediatas atribuidas a este procedimiento, los cuáles están en relación directa al número y tamaño tumoral, y localización de las mismas. Así Collado et al. ${ }^{1}$ reportaron de 2.821 pacientes, 5,1\% de complicaciones, siendo los más frecuentes el sangrado post-operatorio en $2,8 \%$ y perforación vesical en $1,3 \%$, con perforación extraperitoneal en 83\% de los casos e intraperitoneal en el 17\% restante, de los cuáles requirió cirugía abierta el 11\% del total. Se repitió el procedimiento en $2,7 \%$ de los pacientes, siendo la persistencia de la hematuria el motivo en el 84\% de los casos.

Las complicaciones post-operatorias tardías mas temidas son el reflujo vesicoureteral y la estenosis uretral, que se presentan en el 14,9 y $4,7 \%$ respectivamente ${ }^{2}$. Con respecto a este último, su aparición está relacionada con el número de resecciones, siendo del 2,3\% tras la primera, hasta el 7,6\% después de 5 resecciones. También son importantes en su génesis, la dispersión eléctrica y el calibre del instrumental. Otras complicaciones menos frecuentes son la hidronefrosis postRTU y vejiga retráctil.

El método de diagnóstico y seguimiento más fiable es la observación directa, es decir la cistoscopia. Esta puede realizarse con instrumental rígido o flexible, ingresado o ambulatorio. El advenimiento del cistoscopio flexible ha facilitado la tarea de realizarla en régimen de ambulatorio, por la simplicidad del procedimiento y excelente tolerancia del paciente al utilizar un gel lubricante de lidocaina ${ }^{3}$. Estos factores han contribuido a su buena aceptación tanto por parte de los pacientes como de los profesionales.

La visión endoscópica pone de manifiesto los tumores papilares, sólidos e incluso en ocasiones permite sospechar la presencia de CIS, al observar zonas planas hiperémicas, aterciopeladas y de bordes geográficos ${ }^{4}$. Esto ha suscitado la apa- rición de algunas publicaciones como la de Endrizzi et al. ${ }^{5}$, quien encontró una buena correlación entre el diagnóstico cistoscópico y el patológico en muestras de biopsia.

La aparición de la cistoscopia fluorescente, con buenos resultados iniciales, probablemente incrementará la detección del CIS, hasta un 30\% más que la cistoscopia convencional, tal y como lo demuestran publicaciones muy actuales ${ }^{6,7}$.

La historia natural de los tumores urológicos ha determinado que el $70-80 \%$ son superficiales en el momento del diagnóstico, manteniendo la mayoría de ellas esta situación a lo largo de la vida $^{8}$. La tasa de recidiva es del 30-80\% variando en función del grado, estadio, multiplicidad y utilización de terapia adyuvante mientras que la progresiones se situaron en el $4 \%$ de los tumores en estadio Ta y el 30\% de los $\mathrm{T} 1$, variando en relación al grado tumoral dentro de cada estadio(9- AUA pág. 92).

Todo lo anteriormente expuesto nos ha animado a realizar y publicar este trabajo, que a pesar de contar con un número escaso de pacientes, podría servir para la realización de otros.

\section{MATERIAL Y MÉTODOS}

De 112 pacientes diagnosticados y tratados de tumor vesical superficial inicial, entre enero de 1998 y diciembre del 2003, con controles periódicos con cistoscopia, ecografia y citología urinaria, en 13 pacientes se decidió manejar con observación, luego del diagnóstico de recidiva tumoral, hasta la decisión de realizar la resección endoscópica de las mismas. Esta decisión de mantener una conducta expectante se basó en: características clínicas del paciente (edad, estado general, presencia de patologías concomitantes y la presencia o no de síntomas producidas por el tumor), así como en las características propias del tumor (grado y estadio del tumor inicial o de la última recidiva, característica morfológica cistoscópica del tumor recurrente y número de las mismas).

Se decidió realizar los controles con cistoscopia flexible (cistoscopio Olympus $14 \mathrm{Ch}$ ) cada 3 meses, en función de la facilidad de realizarla en forma ambulatoria, en la consulta y con anestesia local, con muy buena tolerancia por parte del paciente. Cabe mencionar además la mutua con- 
fianza que debe existir entre médico y paciente, para que una vez hecho el diagnóstico, plantear al mismo la posibilidad de sólo observación y realizar un tratamiento en cuanto se considere oportuno.

La decisión de seguir con la observación o de realizar una resección transuretral se tomó en base a la variación del aspecto tumoral (aumento de la base de implantación y de la vascularización), aumento del tamaño o número de los tumores y en la presencia o ausencia de síntomas (hematuria o síndrome miccional) o simplemente en la falta de deseo del paciente de continuar con la observación.

En los 13 pacientes señalados, se contabilizaron en total 15 eventos de observación (dos veces en 2 pacientes). Se entiende por un evento, el periodo de observación expresado en meses y que se extiende desde el momento del diagnostico cistoscópico en que se inicia la observación hasta el momento en que se decide la RTU.

Se analizaron las características clínicas y patológicas, constatando la evolución de las mismas.

\section{RESULTADOS}

La edad media de los pacientes fue de 74,6 años (rangos entre 47-91 años). Como se podrá apreciar en la Figura 1 de distribución por edad, la casi totalidad de los enfermos $(84,5 \%)$ estuvieron en los rangos de edad entre 70-79 (4 pacientes) y 80-89 (5 pacientes). En distribución por sexos, 12 pacientes $(92,3 \%)$ correspondieron al sexo masculino y 1 paciente $(7,7 \%)$ al femenino.

La media de tiempo entre el diagnostico del tumor inicial y el del tumor previo al periodo de observación fue de 14,8 meses(rangos 3-29 meses). La media de recidiva previa a la observación fue de 1,76 veces (rangos entre 1-3), y el número total de recidivas, incluidas las de durante y post-observación medió en 2,46 (rangos 1-7).

El tiempo medio de observación fue de 5,76 meses (rangos entre 3-17 meses).

La media de RTU por paciente fue de 3,15 (rangos entre 2-8).

Del total de eventos, en la comparación entre los tumores iniciales y los post-observación, no se observó progresión ni en grado ni en estadio en 11/15 eventos (9 pacientes), representando el

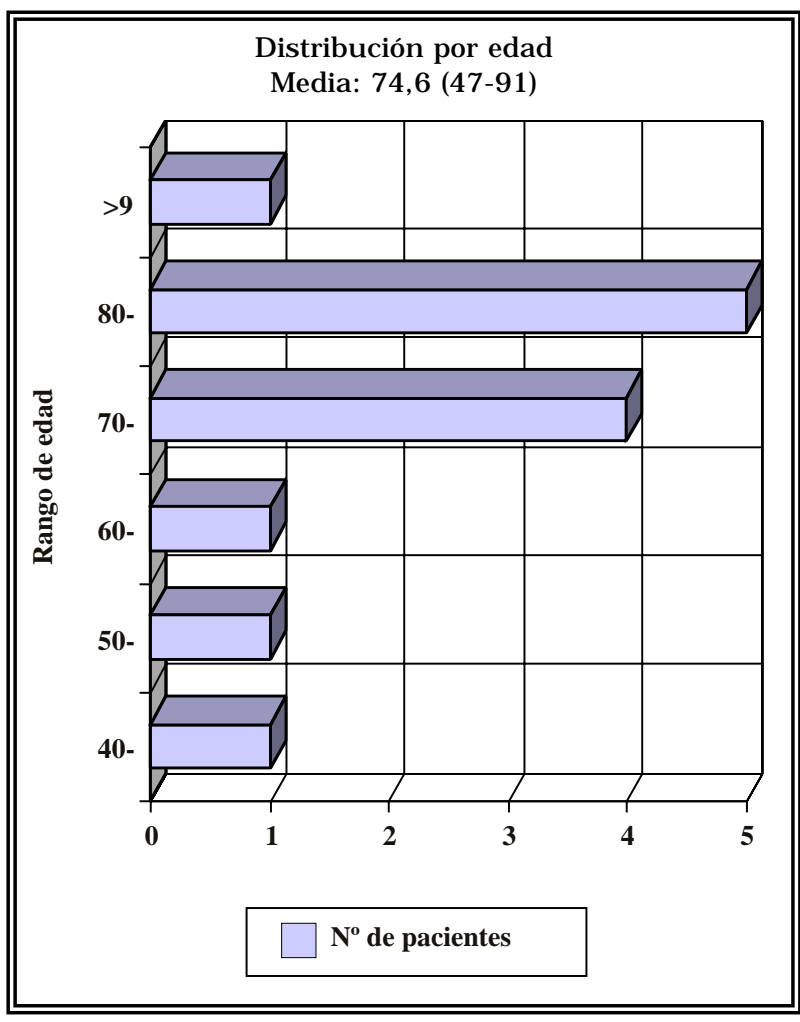

FIGURA 1. Resultados

$73,3 \%$. De los 4 eventos restantes, 2 progresaron en grado y estadio, 1 en grado y 1 en estadio. (Tabla 1).

Entre los tumores previos al periodo de observación y los post-observación, de los 15 eventos, en 12 (10 pacientes) que representa el $80 \%$, no se constató progresión en grado o estadio, aunque en uno de ellos apareción CIS, acompañando a un G3T1. En los 3 restantes, se constató progresión en grado y estadio en 2 y solo de estadio en 1. (Tabla 2).

Tabla 1

Resultados

\begin{tabular}{ccc}
\hline No Ptes. & Tm Inicial & Tm Post-Obs. \\
\hline 2 & G1 Ta & G2 T1 \\
1 & G2 T1 & G3 T1 \\
1 & G2 Ta & G2 T1 \\
1 & G1 Ta & Gx Tx \\
1 & G3 T1 & G3T1 + Cis \\
1 & G2T1 + cis & G1 Ta \\
2 & G2 T1 & G2 Ta \\
2 & G2 Ta & G2 Ta \\
1 & G2 Ta & G1 Ta \\
3 & G1 Ta & G1 Ta \\
\hline
\end{tabular}


Tabla 2

\begin{tabular}{ccc} 
Resultados & & \\
\hline No Ptes. & Tm Pre-Obs. & Tm Post-Obs. \\
\hline 2 & G1 Ta & G2 T1 \\
1 & G2 T1 & G3 T1 \\
1 & G3 T1 & G3T1 + Cis \\
1 & G3 T1 & G3 T1 \\
1 & Gx Tx & G2 Ta \\
1 & G1 Ta & Gx Tx \\
1 & G2 T1 & G2 T1 \\
2 & G2 Ta & G2 Ta \\
1 & G2 T1 & G2 Ta \\
1 & G2 Ta & G1 Ta \\
3 & G1 Ta & G1 Ta
\end{tabular}

En ningún caso se constató progresión a tumor vesical infiltrante ni aparición de tumor en tramo urinario superior.

\section{DISCUSIÓN}

A pesar de que en un trabajo reciente, realizado con un buen número de pacientes, en que no encontraron correlación entre la visión endoscópica y el hallazgo anatomopatológico consideramos contraproducente el hecho de la participación de numerosas personas, tanto para el diagnóstico como el tratamiento, sobretodo teniendo en cuenta la subjetividad de los hallazgos cistoscópicos y la variabilidad de la técnica operatoria ${ }^{10}$. Esta situación nosotros la obviamos ya que los procedimientos fueron realizados en su totalidad por 2 urólogos y la revisión histopatológica, tanto de los tumores iniciales como los recidivados fueron hechos siempre por el mismo profesional.

Soloway et al. ${ }^{11}$ reportaron asimismo un total de 32 pacientes manejados con conducta expectante, incluyendo tumores con aspecto cistoscópico de bajo riesgo de progresión (todos con historia previa de tumores Ta o T1 y tumores Ta previos a la observación). La edad media de los pacientes fue de 72 años (rangos 39-88) con poca variación con respecto a los incluidos en nuestro estudio cuya media fue de 74,6 años. La elevada media de edad se explica, ya que incluimos en este manejo a pacientes con varias resecciones anteriores y patologías concomitantes, que normalmente aumentan la morbilidad intra y post- operatorio. Ellos han comunicado una progresión de $6,7 \%$ de G1 a G2 y de Ta a T1. En contrapartida, nosotros hemos encontrado una progresión global del 20\% (3 de 15 eventos) y la aparición de CIS en un paciente con G3T1 previo. Evidentemente los resultados de progresión no son comparables, ya que nosotros incluimos tumores de todos los grados y estadios superficiales, a pesar del cuál no hemos constatado ninguna progresión a tumor infiltrante ni aparición de tumor en tramo urinario superior.

No hemos querido entrar en detalles con respecto a la utilización de terapia adyuvante ya que no constituye el propósito de este estudio.

La utilidad de la cistoscopia flexible tal y como lo hemos mencionado anteriormente, radica además en que hace posible la fulguración ambulatoria y en la consulta de tumores igual o menor a $5 \mathrm{~mm}$ y de aspecto papilar y bajo grado ${ }^{12}$. Conste que nos parece una actitud válida ésta alternativa no la hemos adoptado en vista de que incluimos tumores de mayor grado y estadio y por otro lado decidimos la resección para obtener material para anatomía patológica.

Considerando los dos últimos comentarios analizados, tanto la conducta expectante en los tumores vesicales recidivados o la fulguración ambulatoria, por separado o más si se considera conjuntamente sería de mucha utilidad en pacientes añosos, con múltiples patologías acompañantes, mal estado general o con recidivas frecuentes de aspecto superficial y bajo grado. Esto ayudaría a disminuir la morbimortalidad atribuible a la RTU y potencialmente mejoraría la calidad de vida de los pacientes, teniendo además un impacto económico en el coste sanitario.

\section{CONCLUSIÓN}

Debido a la mínima proporción de progresión, ninguna a tumor infiltrante y a la escasa manifestación clínica, consideramos que las recidivas podrían ser manejadas durante un tiempo con observación, sobretodo en los casos de tumores de bajo grado o estadio, disminuyendo así la morbilidad y el coste asociados a la resección. Además la valoración cistoscópica y anatomopatológica, deberían ser realizadas por el mismo urólogo y patólogo respectivamente, a fin de reducir la variación inter examinador. 


\section{Aclaraciones}

CIS: Carcinoma in situ. TVS: Tumor vesical superficial. TUS: Tumor de tramo urinario superior. RTU: Resección transuretral. № Ptes: Número de pacientes. Tm inicial: Tumor inicial. Tm Pre-Obs.: Tumor pre-observación. Tm PostObs.: Tumor post-observación.

\section{REFERENCIAS}

1. Collado A, Chéchile G, Salvador J, Vicente J. Early complications of endoscopic treatment for superficial bladder tumors. J Urol 2000;164:1529-1532.

2. Vicente Rodríguez J. Tratamiento endoscópico. En "Tumores vesicales superficiales". J Vicente, G Chéchile, J Salvador. (eds) Acción Médica. Madrid, 2000;121-143.

3. Herr HW, Schneider M. Outpatient flexyble cystoscopy in men: a randomized study of patient tolerance. J Urol 2001; 165:1971-1972.

4. Vicente J, Algaba F. Carcinoma vesical. En "Semiologia diagnóstica endovesical”. Pulso ediciones. Barcelona, 1998;152168.

5. Endrizzi J, Cina S, Hartmon W et al. Correlation of cistoscopic impresión and histologic diagnosis on biopsy specimens of the bladder. J Urol 2000;163:134(A588).

6. Jichlinski P, Marti A, Leisinger HJ. Fluorescence cystoscopy in bladder cancer: what future?. Ann Urol 2002;36:264268.

7. Schmidbauer J, Witjes F, Shmeller N, Donat R, Susani M, Marberger M, and Members of the Hexvix Pcb301/01 Study
Group. Improved detection of urothelial carcinoma in situ with hexaminolevulinate fluorescence cystoscopy. J Urol 2004;171:135-138

8. Martínez Sarmiento M, Vera Donoso CD, Jiménez Cruz JF. Diagnóstico y protocolos de seguimiento en la recidiva del tumor vesical superficial. En "Diagnóstico y tratamiento de las recidivas en los tumores urológicos". J. Fernando Jiménez Cruz, C.D. Vera Donoso. Grupo Aula Médica. Madrid. 1996;75-87.

9. El-Gabry EA, Strup SE, Gomilla LG. El cáncer superficial de vejiga: epidemiología, diagnóstico e historia natural. En "AUA update Series. Edición española 2001;1:85-108.

10. De León Morales E, Arango Toro O, Lorente Marín JA, Cortadellas Ángel R, Bielsa Gali O, Gelabert Mas A. La impresión cistoscópica frente al diagnóstico histológico en los tumores vesicales. ¿coinciden?. Actas Urol Esp 2003; 27(1):18-21.

11. Soloway MS, Bruck DS, Kim SS. Expectant management of small, recurrent, noninvasive papillary bladder tumors. J Urol 2003;170(2Pt1):438-441.

12. Donat SM, North A, Dalbagni G, Herr HW. Efficacy of office fulguration for recurrent low grade papillary bladder tumors less than 0,5 cm. J Urol 2004;171(2Pt1):636-639.

Dr. P. Martínez Cáceres

Dpto. de Urología. Instituto Médico Tecnológico

Passeig D’Amunt no $14,2^{\circ}$ piso

08024 Barcelona

(Trabajo recibido el 13 enero 2005) 